\title{
The black hole in NGC 1313 X-2
}

\author{
Luca Zampieri $^{1, \star}$ and Alessandro Patruno ${ }^{2}$ \\ 1 INAF-Osservatorio Astronomico di Padova, Padova I-35122, Italy \\ 2 Astronomical Institute A. Pannekoek, University of Amsterdam, Science Park 904, 1098 XH Amsterdam, The Nether- \\ lands
}

Received , accepted

Published online

\begin{abstract}
Key words galaxies: NGC 1313 - stars: individual (NGC 1313 X-2) - X-rays: binaries - X-rays: galaxies - X-rays: individuals (NGC 1313 X-2)

The amount of data available for NGC 1313 X-2 make it a cornerstone for the study of ultraluminous X-ray sources (ULXs). We modelled the optical and X-ray data of this ULX with a binary evolution code that takes into account X-ray irradiation. We restricted the candidate binary system to be either a 50-100 $M_{\odot}$ black hole $(\mathrm{BH})$ accreting from a $12-$ $15 M_{\odot}$ main sequence star or a $20 M_{\odot} \mathrm{BH}$ with a $12-15 M_{\odot}$ giant donor. If the orbital period of the system is $\sim 6$ days, a $20 M_{\odot} \mathrm{BH}$ model becomes unlikely and we are left with the only possibility that the compact accretor in NGC1313 X-2 is a massive $\mathrm{BH}$ of $50-100 M_{\odot}$. We briefly discuss these results within the framework of an alternative scenario for the formation of ULXs, in which a portion of them may contain BHs of $\gtrsim 30-40 M_{\odot}$ formed from very massive stars in a low metallicity environment.
\end{abstract}

(C) WILEY-VCH Verlag GmbH \& Co. KGaA, Weinheim

\section{Introduction}

Several pieces of observational evidence strongly suggest that a large fraction of ULXs are accreting black hole X-ray binaries with massive donors (see, e.g., Zampieri \& Roberts 2009 and references therein). Their X-ray spectral properties, along with the long-term flux variability, are similar to those observed in Galatic X-ray binaries although, in the last few years, Chandra and XMM-Newton observations revealed new behaviours, showing the existence of spectral states with rather peculiar properties (e.g. Gladstone et al. 2009). At the same time, while most ULXs appear to show little variations on timescales of seconds to hours (e.g. Heil et al. 2009), in some cases fast X-ray variability is reminiscent of the behaviour observed in Galactic accretionpowered sources (rms variability, broad band noise, quasi periodic oscillations; e.g. Strohmayer \& Mushotzky 2003, Strohmayer et al. 2007, Feng et al. 2010, Heil \& Vaughan 2010). Probably, the most compelling evidence of the binary nature of ULXs is provided so far by the detection of a modulation in the X-ray light curve of M $82 \mathrm{X}-1(\sim 62$ days; Kaaret et al. 2006) and NGC 5408 X-1 ( 115 days; Strohmayer 2010), that are interpreted as the orbital periods of these systems.

A number of ULXs are associated to stellar optical counterparts and some of them have been identified with stars of known spectral type (e.g. Liu et al.2002,2004, Kaaret et al. 2004, Mucciarelli et al. 2005, Soria et al. 2005). The counterparts appear almost ubiquitously hosted in young stellar

\footnotetext{
* Corresponding author: e-mail: luca.zampieri@oapd.inaf.it
}

environments (e.g. Pakull et al. 2006, Ramsey et al. 2006 Liu et al. 2007) and have properties consistent with those of young, massive stars. NGC $1313 \mathrm{X}-2$ is one of the few ULXs with a well identified optical counterpart. It is located $\sim 6$ ' south to the nucleus in the barred spiral galaxy NGC 1313 at a distance of 3.7-4.27 Mpc (Tully 1988, Méndez et al. 2002, Rizzi et al. 2007). Its observed X-ray luminosity varies between a few $\times 10^{39} \mathrm{erg} \mathrm{s}^{-1}$ and $\sim 10^{40} \mathrm{erg} \mathrm{s}^{-1}$ in the $0.3-10 \mathrm{keV}$ band (Feng \& Kaaret 2006, 2007). The large amount of data available make this source a cornerstone for the study of ULXs.

\section{A bit of history: X-rays}

NGC 1313 X-2 was discovered by Einstein (Fabbiano \& Trinchieri 1987) and included in the Einstein Extended Medium Sensitivity Survey as MS 0317.7-6647. Stocke et al. (1995) originally proposed that it could be either a Galactic isolated neutron star or a binary containing a massive $\mathrm{BH}$ in NGC 1313.

It was then repeteadly observed with other satellites, including ROSAT (1991-1998; Stocke et al. 1995, Colbert et al. 1995, Miller et al. 1998, Schlegel et al. 2000), ASCA (1993-1995; Petre et al.1994, Makishima et al.2000), Chandra and XMM-Newton (2000-2006; Zampieri et al. 2004, Feng \& Kaaret 2005 and references therein). The X-ray spectrum of NGC 1313 X-2 can be modelled in different ways and shows the typical features shared by other bright ULXs, such as the presence of a soft, thermal component and a rollover of the spectrum above $\sim 3 \mathrm{keV}$ (e.g. Gladstone et al.2009, Stobbart et al.2006 and references therein). 
The amount of XMM-Newton spectra now available allow for a tentative identification of different spectral states (see Pintore \& Zampieri, these Proceedings).

\section{A bit of history: optical}

The optical counterpart of NGX 1313 X-2 was first identified on an ESO $3.6 \mathrm{~m} R$ band image thanks to an accurate Chandra astrometry and after registering the X-ray image on the position of SN 1978K (Zampieri et al. 2004). ESO VLT images of the field showed that the counterpart was actually composed of two distinct objects (C1 and C2), separated by $\sim 0.7$ " (Mucciarelli et al. 2005). Further refinement in the astrometry and accurate modelling of the optical emission indicated that object C1 was the more likely counterpart (Liu et al. 2007, Mucciarelli et al. 2007, Patruno \& Zampieri 2008). However, this was established beyond any doubt by the detection of the $4686 \mathrm{He}$ II emission line in its optical spectrum, a characteristic imprint of X-ray irradiation (Pakull et al.2006, Grisé et al.2008).

The stellar environment of NGC 1313 X-2 has also provided interesting constraints. There are two groups of young stars spread out over $\sim 200$ pc. Isochrone fitting of the colour-magnitude diagram of these groups has been attempted and provides cluster ages of 20 \pm 5 Myrs (Pakull et al.2006. Ramsey et al. 2006, Liu et al. 2007, Grisé et al. 2008). As several other ULXs, NGC 1313 X-2 is also associated with a very extended $(\sim 400 \mathrm{pc})$ optical emission nebula that gives important information on the energetics and lifetime of the system (Pakull \& Mirioni 2002). Assuming that it is formed in an explosive event or that its mechanical energy comes from the ULX wind/jet activity, the characteristic energy and age of the nebula turn out to be $\sim 10^{52}-10^{53} \mathrm{erg}$ and $\sim 1$ Myr, respectively (Pakull et al. 2006). Finally, several different estimates of the mass and luminosity class of the optical counterpart of NGC $1313 \mathrm{X}-2$ were reported in the literature (see e.g. Patruno \& Zampieri 2010 and references therein).

Recently, Liu et al. (2009) found a possible periodicity of $6.12 \pm 0.16$ days in the $\mathrm{B}$ band light curve of the optical counterpart of NGC $1313 \mathrm{X}-2$, that was interpreted as the orbital period of the binary system. Three cycles were detected in the $B$ band, while no modulation was found in $V$. Previous studies carried out on the available HST and VLT observations led to negative results (Grisé et al.2008). More recently, lack of significant photometric variability on a new sequence of VLT observations has been reported by Grisét al. (2009). In principle, the detection of the orbital period would definitely confirm the identification of the optical counterpart and the binary nature of this system. Most importantly, it would open the way to perform a dynamical measurement of the $\mathrm{BH}$ mass.

\section{Optical constraints}

In the following we use the X-ray luminosity and all the available optical data on NGC $1313 \mathrm{X}-2$ to constrain the properties of this ULX and its $\mathrm{BH}$. This is probably one of the most accurate analyses that is possible to perform at present on a single ULX. We adopt the $V$ and $B$ band photometry of the counterpart as determined by Mucciarelli et al.2007. As the source is variable, we have further corrected the magnitudes and colours by using the average value of $V$ and by propagating the errors on $V$ and $B$. The error in the absolute magnitudes is taken to be equal to the maximum uncertainty in the different distance determinations of NGC 1313 (Tully 1988, Méndez et al.2002, Rizzi et al.2007).

Concerning the reddening, two different estimates of the colour excess were adopted in the literature: $E(B-V)=$ 0.1 (2007, Grisé et al. 2008) and $E(B-V)=0.3$ (Liu et al. 2007). In the following we chose $E(B-V)=0.11$. If we assume $E(B-V)=0.3$, it is not possible to obtain agreement between the binary evolution models and all the simultaneous constraints coming from observations. For $E(B-V)=0.11$ the adopted absolute $V$ magnitude is therefore in the range -4.38 to -4.79 and the $B-V$ colour is $-0.13 \pm 0.06$.

Three different values of the metallicity inferred from an abundance analysis of the HII regions in NGC 1313, all sub-solar, are reported in the literature: $Z=0.1 Z_{\odot}(\mathrm{Pi}-$ lyugin 2001), $Z=0.2 Z_{\odot}(\operatorname{Ryder} 1993)$ and $Z=0.4 Z_{\odot}$ (Walsh \& Roy 1997, Hadfield \& Crowther 2007). Therefore, although the metallicity in the environment of NGC 1313 X-2 appears to be definitely sub-solar, the actual value is still uncertain (see Ripamonti et al., these Proceedings; see also Pintore \& Zampieri, these Proceedings, for an independent metallicity estimate from X-ray spectra).

Finally, we re-analyzed the optical photometry of NGC $1313 \mathrm{X}-2$ with the aim of clarifying the statistical significance of the orbital periodicity identified by Liu et al. (2009). As reported in Impiombato et al. (these Proceedings), the folded $B$ band light curve shows a 6 days periodicity with a significance slightly larger than $3 \sigma$. This suggests that the periodicity may be there, but the low statistical significance of the $B$ band modulation, along with the lack of detection in the $V$ band, make its identification uncertain.

\section{Modeling the NGC 1313 X-2 binary system}

We modelled NGC 1313 X-2 using the binary evolution code described in Patruno \& Zampieri (2008), that computes the evolution of the orbital parameters and the companion star taking into account the contribution of the accretion disc and X-ray irradiation during the contact phases. For this analysis, we developed a new version of the code, with a more accurate calculation of the optical colours (Patruno \& Zampieri 2010).

The evolution is computed using two different values of the (zero age) metallicity, both sub-solar: $Z=0.2 Z_{\odot}$ 


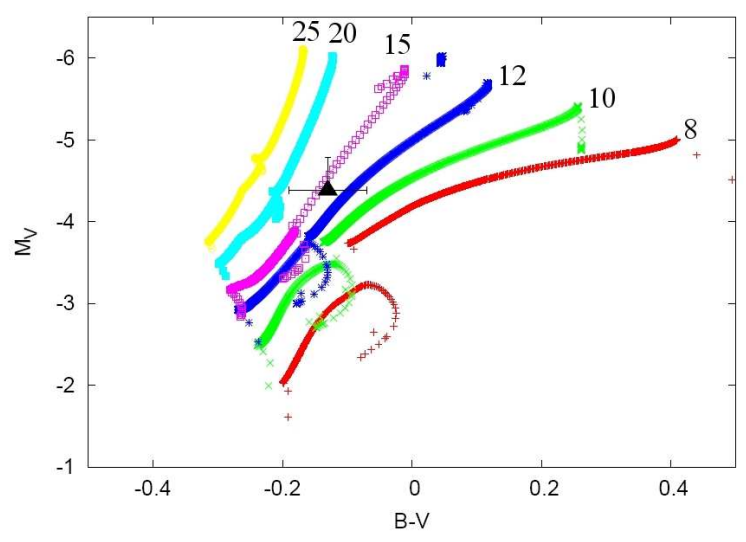

Fig. 1 CM diagram for binaries with a $20 M_{\odot} \mathrm{BH}$ undergoing case $A B$ mass transfer. The black triangle marks the position of the optical counterpart of NGC $1313 \mathrm{X}-2$. All the tracks are plotted only during the contact phases. The lower part of each track (up to the turn around) refers to the MS RLOF phase, whereas the upper part to the H-shell burning RLOF phase. The labels on each curve indicate that mass of the donor star (in $M_{\odot}$ ).

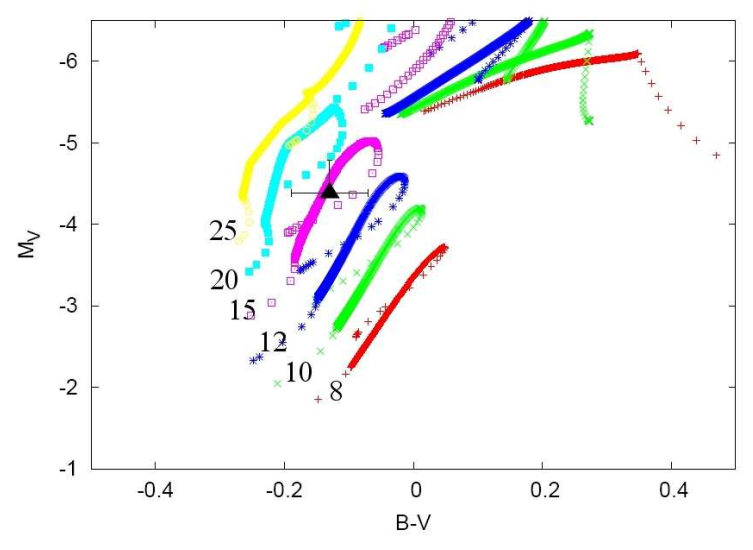

Fig. 2 Same as Figure 1 for a $70 M_{\odot} \mathrm{BH}($ case $A B)$.

and $Z=0.5 Z_{\odot}$. We found that the effects on the evolutionary tracks and the stellar colours are minimal. Hence, for reference, in the following we will consider only tracks computed for $Z=0.5 Z_{\odot}$.

If the donor starts a contact phase via Roche-lobe overflow (RLOF), we assume that a standard accretion disc forms around the $\mathrm{BH}$. The accretion rate is instantaneously taken to be equal to the mass-transfer rate from the companion. When the accretion rate $\dot{M}$ exceeds the Eddington rate $\dot{M}_{E d d}$, we impose $\dot{M}=\dot{M}_{E d d}$ and assume that the excess mass is expelled from the system. In our simulations, all the binaries that start RLOF on the main sequence (MS) have also a second episode of mass transfer after the terminal age main sequence (TAMS). Following Patruno \& Zampieri (2010), we term such binary models as Case $A B$, while those starting the first contact phase after the TAMS are termed Case $B$.
For the latter, typically the contact phase is too short when compared to the characteristic age of the nebula ( $\sim 1$ Myrs), which provides an estimate of the active phase of the ULX. Therefore, from now on, we will focus on Case $A B$ systems.

\subsection{Evolution of a binary with a $20 M_{\odot}$ BH}

Figure 1 shows the evolutionary tracks on the color-magnitude (CM) diagram computed for binary systems with a $\mathrm{BH}$ of $20 M_{\odot}$ and donors of different masses. Considering the intrinsic variability of the counterpart, its position on the CM diagram may be broadly in agreement with $10-20 M_{\odot}$ donors during $\mathrm{H}$-shell burning. However, only stars in the $12-15 M_{\odot}$ have characteristic ages consistent with the estimated parent cluster age (15-25 Myrs). Donors this massive, during $\mathrm{H}$-shell burning, provide a mass transfer rate largely above Eddington. In this case there is excess mass that must be somewhat expelled from the system. Beaming and/or super-Eddington emission is necessary to explain the observed ULX luminosity. We note also that the system certainly underwent a first contact phase during MS, during which it had time to energize the surrounding nebula.

\subsection{Evolution of a binary with a $70 M_{\odot} \mathbf{B H}$}

Figure 2] shows the evolutionary tracks on the CM diagram for a systems with a $\mathrm{BH}$ of $70 M_{\odot}$ and donors of different masses. The position of the optical counterpart is now consistent with the tracks of stars on the MS, in the mass range between 12 and $20 M_{\odot}$. Again, the further constrain inferred from the parent cluster age (15-25 Myrs) restricts the allowed donor masses to the interval $12-15 M_{\odot}$. The mass transfer of a $15 M_{\odot}$ at this stage is typically between $2 \times 10^{-7}$ and $2 \times 10^{-6} M_{\odot} \mathrm{yr}^{-1}$, consistent with the accretion rate $\left(\sim 10^{-6} M_{\odot} \mathrm{yr}^{-1}\right)$ inferred from the measured average luminosity of NGC $1313 \mathrm{X}-2\left(\sim 4 \times 10^{39} \mathrm{erg}\right.$ $\mathrm{s}^{-1}$; Mucciarelli et al. 2007). Similar results are obtained for BHs with a mass between 50 and $100 M_{\odot}$.

\subsection{Evolution of the orbital period}

The evolution of the orbital period $P$ is rather different for the two systems. In order to have a first contact phase during MS, the initial orbital separation is $\sim 1$ day. For the $20 M_{\odot} \mathrm{BH}$ binary, the orbital period at the end of MS is $\sim 4$ days. During the $\mathrm{H}$-shell burning phase it increases rapidly up to 20 days. When the $12-15 M_{\odot}$ donor tracks cross the position of the counterpart on the $\mathrm{CM}$ diagram, $P \gtrsim 8$ days.

For the $70 M_{\odot} \mathrm{BH}$ binary, the orbital period at TAMS reaches $\sim 6$ days and is between 5.5 and 6.5 days when the evolutionary tracks of the $12-15 M_{\odot}$ donors are consistent with the photometric properties of the optical counterpart.

\section{Discussion}

According to the actual value of the orbital period of the system and considering the constraints from the inferred mass 
transfer rate, the position on the CM diagram, the characteristic ages of the parent stellar cluster and the bubble nebula, the following scenarios are possible:

$-P \lesssim 4$ days: mass transfer from a $\sim 15 M_{\odot}$ MS donor onto $\mathrm{a} \sim 20 M_{\odot} \mathrm{BH}$, if all the mass is accreted onto the $\mathrm{BH}$ and super-Eddington emission is allowed.

- $P=5-7$ days: mass transfer of a MS donor of $12-$ $15 M_{\odot}$ onto a $50-100 M_{\odot} \mathrm{BH}$.

- $P \gtrsim 8-10$ days: mass transfer of a $\mathrm{H}$-shell burning donor of $12-15 M_{\odot}$ onto a $\sim 20 M_{\odot}$ BH. If $P \sim 12$ days, the photometry is consistent with the possition of the counterpart only for donors of $\sim 20 M_{\odot}$, too young with respect to the age of the parent cluster.

- $P \gtrsim 15-20$ days: mass transfer of a H-shell burning donor of $\sim 15 M_{\odot}$ onto a $\sim 20 M_{\odot} \mathrm{BH}$, only if $\mathrm{X}$ ray irradiation is switched off (because, e.g., emission is beamed and not able to hit the disc and donor surfaces).

If we use the tentative identification of the $\sim 6$ days orbital period originally reported by Liu et al. (2009) as a further constraint, the observational data appear to be consistent only with the second scenario and we are left with the only possibility that the compact accretor in NGC 1313 $\mathrm{X}-2$ is a massive black hole of $50-100 M_{\odot}$.

Such a system would fit well within a massive BH scenario for ULXs forming in a low-metallicity natal environment (Mapelli et al.2009, Zampieri \& Roberts 2009, Mapelli et al.2010; Mapelli et al., these Proceedings). Albeit uncertain, in fact, the metallicity of the environment of NGC 1313 $\mathrm{X}-2$ is definitely sub-solar. As mass loss becomes less efficient in such environments, already at $Z \lesssim 0.2-0.3 Z_{\odot}$ massive stars may retain a significant fraction of their envelopes at the end of their life (see e.g. Zampieri \& Roberts 2009 and references therein). If the envelope is more massive than $\sim 30-40 M_{\odot}$, a low metallicity star may collapse directly to form a $\mathrm{BH}$ (Heger et al. 2003. Belczynski et al. 2009). This occurs because, after core collapse, the supernova shock wave loses too much energy in trying to unbind the envelope until it stalls and most of the star collapses into a $\mathrm{BH}$ of mass comparable to the star final mass ( $30-40 M_{\odot}$; Fryer 1999. Zampieri 2002). With these massive BHs only very modest beaming or slight violations of the Eddington limit (a factor of a few) are needed to account for the luminosity of bright ( $~\left(10^{40} \mathrm{erg} \mathrm{s}^{-1}\right.$ ) ULXs.

As noted above, however, the identification of the orbital period of NGC $1313 \mathrm{X}-2$ remains uncertain. Furthermore, we did not perform a complete survey of the parameter space evolving case $A B$ systems with $\mathrm{BH}$ masses between 20 and $50 M_{\odot}$. For $\mathrm{BH}$ masses in this range there may also be agreement with observations with other values of $P$. Finally, modelling the amplitude and shape of the light curve (considering X-ray irradiation and ellipsoidal modulations) may give additional constraints. A systematic investigation of this type is postponed to when a more robust assessment of the orbital period will be available.

Acknowledgements. LZ acknowledges financial support through INAF grant PRIN-2007-26. We thank the referee for useful com- ments. AP acknowledges support from the Netherlands Organization for Scientific Research (NWO) Veni Fellowship.

\section{References}

Belczynski, K., et al. : 2009, ApJ 714, 1217

Colbert, E. J. M., et al.: 1995, ApJ 446, 177

Fabbiano, G., Trinchieri, G.: 1987, ApJ 315, 46

Feng, H., Kaaret, P.: 2005, ApJ 633, 1052

Feng, H., Kaaret, P.: 2006, ApJ 650, 75

Feng, H., Rao, F., Kaaret, P.: 2010, ApJ 710, L137

Fryer, C. L.: 1999, ApJ 522, 413

Gladstone, J. C., Roberts, T. P., Done, C.: 2009, MNRAS 397, 1836

Grisé F., et al.: 2008 , A\&A 486, 151

Hadfield, L. J., Crowther, P. A.: 2007, MNRAS 381, 418

Heger, A., et al.: 2003, ApJ 591, 288

Heil, L.M., Vaughan, S.: 2010, MNRAS, in press (arXiv:1003.5825)

Heil, L. M., Vaughan, S., Roberts, T. P.: 2009, MNRAS 397, 1061

Kaaret, P., Ward, M. J., Zezas, A.: 2004, MNRAS 351, L83

Kaaret, P., Simet, M. G., Lang, C. C.: 2006, Science 311, 491

Liu, J., Bregman, J. N., Seitzer, P.: 2002, ApJ 580, L31

Liu, J. et al.: 2004, ApJ 602, 249

Liu, J. et al.: 2007, ApJ 661, 165

Liu, J. et al.: 2009, ApJL 690, L39

Makishima, K., et al.: 2000, ApJ 535, 632

Mapelli, M., Colpi, M., Zampieri, L.: 2009, MNRAS 395, L71

Mapelli, M., et al.: 2010: MNRAS, in press (arXiv1005.3548)

Méndez, B., et al.: 2002, AJ 124, 213

Miller, S., et al.: 1998, AJ 116, 1657

Mucciarelli, P. et al.: 2005, ApJL 633, L101

Mucciarelli, P. et al.: 2007, ApJ 658, 999

Pakull, M. W., Mirioni, L.: 2002, preprint (astro-ph/0202488)

Pakull, M.W. et al.: 2006, IAU Symp. 230, 293

Patruno, A., Zampieri, L.: 2008, MNRAS 386, 543

Patruno, A., Zampieri, L.: 2010, MNRAS 403, L69

Petre, R., et al.: 1994, PASJ 46, L115

Pilyugin L. S.: 2001, A\&A 369, 594

Ramsey, C.J. et al.: 2006, ApJ 641, 241

Rizzi, L., et al.: 2007, ApJ 661, 815

Ryder, S. D.: 1993, Ph.D. Thesis

Schlegel, E. M., et al.: 2000, AJ 120, 2373

Soria, R., et al.: 2005, MNRAS 356, 12

Stobbart A.-M., Roberts T. P., Wilms J.: 2006, MNRAS 368, 397

Stocke, J. T., et al.: 1995, AJ 109, 1199

Strohmayer, T. E. Mushotzky, R. F.: 2003, ApJ 586, L61

Strohmayer, T. E., et al.: 2007, ApJ 660, 580

Strohmayer, T. E.: 2010, ApJL, in press (arXiv:0911.1339

Tully, R. B.: 1988, Nearby Galaxies Catalog. Cambridge Univ. Press, Cambridge

Walsh, J. R., Roy, J.-R.: 1997, MNRAS 288, 726

Zampieri, L., 2002: in Cianci R., Collina R., Francaviglia M., Fre P., eds, 14th SIGRAV Conference on General Relativity and Gravitational Physics. Springer, Milan, 301

Zampieri, L. et al.: 2004, ApJ 603, 523

Zampieri, L., Roberts, T. P.: 2009, MNRAS 400, 677 